\title{
Correction to "Evaluation of Operational Models of Agonism and Allosterism at Receptors with Multiple orthosteric Binding Sites"
}

In the above article [Gregory KJ, Giraldo J, Diao J, Christopoulos A, and Leach K (2020) Mol Pharmacol, 97(1): 35-45; DOI: https://doi.org/10.1124/mol.119.118091], the appendix section was accidentally omitted. The HTML and PDF versions of the article have been updated to include this information.

The compositor regrets this error and any inconvenience it may have caused. 